\title{
Anabases
}

ANABASES Traditions et réceptions de l'Antiquité

\section{Hédelin François, abbé D’AUBIGNAC, Conjectures académiques ou Dissertation sur l'Iliade}

Sylvie Rougier-Blanc

\section{OpenEdition}

\section{Journals}

Édition électronique

URL : http://journals.openedition.org/anabases/2084

DOI : 10.4000/anabases. 2084

ISSN : 2256-9421

\section{Éditeur}

E.R.A.S.M.E.

\section{Édition imprimée}

Date de publication : 1 mars 2011

Pagination : 289-291

ISSN : 1774-4296

\section{Référence électronique}

Sylvie Rougier-Blanc, « Hédelin François, abbé D'AuBıgnac, Conjectures académiques ou Dissertation sur I'lliade », Anabases [En ligne], 13 | 2011, mis en ligne le 01 novembre 2011, consulté le 22 septembre 2020. URL : http://journals.openedition.org/anabases/2084 ; DOI : https://doi.org/10.4000/anabases 2084

Ce document a été généré automatiquement le 22 septembre 2020

(c) Anabases 


\title{
Hédelin François, abbé D’AUBIGNAC, Conjectures académiques ou Dissertation sur l'Iliade
}

\author{
Sylvie Rougier-Blanc
}

\section{RÉFÉRENCE}

Hédelin François, abbé D’AUBIGNAC, Conjectures académiques ou Dissertation sur l'Iliade, éd. critique, avec une introduction, des notes et une conclusion par Gérard Lambin, coll. "Sources classiques », $\mathrm{n}^{\circ}$ 101, Paris, Honoré Champion, 2010, 352 p. 60 euros / ISBN 978-2-7453-2028-5.

1 L'édition critique que propose Gérard Lambin de la Dissertation sur l'Iliade de François Hédelin dit l'abbé d'Aubignac offre un nouvel instrument de travail pour qui étudie l'histoire de la question homérique. L'abbé d'Aubignac est en effet le premier à avoir ouvertement nié l'existence d'Homère et formulé l'hypothèse d'une œuvre composite, résultat du travail de nombreux compilateurs à des époques différentes, et ce en pleine querelle des Anciens et des Modernes, au moment même où s'opposaient partisans et pourfendeurs d'Homère (probablement dans les années 1660). L'abbé d'Aubignac a ouvert la voie de la théorie des chants successifs des analystes mais son œuvre, publiée longtemps après sa mort, en 1715 , au moment où la Querelle perdait de sa vigueur, a été oubliée puis copiée. Imprimées bien tardivement et tombées dans l'oubli, les Conjectures ont été plagiées par F. A. Wolf qui passa longtemps pour l'inventeur de la théorie des chants successifs, comme l'avait bien analysé au début du siècle dernier V. Bérard (dans Un mensonge de la science allemande. Les Prolégomènes à Homère de Frédéric-Auguste Wolf, Paris, 1917). Le propos de G. Lambin (à qui l'on doit Homère, le compagnon, Paris, CNRS, 1995) dans cette édition critique des Conjectures est en quelque sorte de réhabiliter l'œuvre comme l'auteur. 
2 L'ouvrage est composé d'une introduction et d'une conclusion (près d'une centaine de pages pour la première, une cinquantaine pour la seconde) ajoutées au texte lui-même, édité selon la méthode scientifique la plus rigoureuse : confrontation des différentes éditions, notes de correction du texte, maintien en ajout entre crochets droits de la pagination originale, respect scrupuleux de l'orthographe originelle, très souvent incohérente et/ou fautive, autant de points qui n'avaient pas été retenus dans la réédition de 1925 due à Victor Magnien. Ces choix éditoriaux motivent l'interprétation de l'œuvre: les nombreuses négligences de forme expliquent en partie le peu d'écho des Conjectures. Écrites trop vite, sous la dictée, comme en témoignent les graphies fantaisistes de noms propres, publiées sans le contrôle de l'auteur, et probablement sans relecture de Germain Brice ni de François Fournier, à l'origine de l'édition, les Conjectures n'ont que très inégalement marqué les savants du Siècle des lumières. Ce volume permet donc de restituer non seulement la pensée de l'auteur mais surtout les faiblesses de l'édition posthume et d'expliquer le dédain qu'a connu l'œuvre. Il donne enfin au lecteur les moyens de remettre dans le contexte de la publication la fameuse Dissertation sur l'Iliade. Car non seulement on se lassait de la querelle d'Homère en ce début de $\mathrm{XVIII}^{\mathrm{e}}$ s., mais encore le temps des publications plus sérieuses (comme le premier volume de l'Iliad d'Alexander Pope) était venu. Dans un tel contexte, on comprend que les Conjectures soient passées relativement inaperçues.

3 Les Conjectures ont été publiées trop tard ou trop tôt, et surtout mal. L'abbé d'Aubignac, dont G. Lambin retrace la vie et la carrière dans l'introduction, fut l'inventeur maladroit de la question homérique, mais il présente une certaine forme de probité intellectuelle surprenante pour un religieux. Il ne se livre jamais à une lecture allégorique de l'Iliade. Il utilise systématiquement la méthode inductive, avec des élans de rigueur admirables pour son temps. Les Conjectures commencent par un éloge de l'ordre et $\mathrm{du}$ respect des règles dans le but d'excuser les propos peu orthodoxes développés dans la suite de l'ouvrage. Et en même temps l'auteur se défend de ne pas critiquer assez ouvertement Homère : il va sans dire qu'il cherche ainsi à se démarquer de la Querelle pour donner à ses idées une portée plus large que la simple polémique entre Anciens et Modernes; on sent même des prétentions (certainement vaines) à l'objectivité et une forme d'humilité (il renonce à traiter de l'odyssée, texte plus complexe). Mais on doit surtout à l'abbé d'Aubignac d'oser s'insurger contre la complaisance d'habitude, contre l'argument d'autorité qui prévaut sur « la lumière de la connaissance » (p. 7). L'articulation de l'ensemble du texte est aussi très rigoureuse et si G. Lambin en transmet un bref plan en fin d'introduction (p. 85-89), très clair, il aurait peut-être été utile de rappeler l'articulation dans le texte même, pour mieux guider le lecteur. Mais il est vrai que le parti pris est de nous fournir le texte tel qu'il fut publié en 1715. Après une série de remarques générales, Aubignac développe les arguments internes puis externes en faveur de sa théorie. Il s'appuie tout d'abord sur la divergence des sources sur l'origine et l'identité d'Homère pour remettre en cause son existence. Il développe ensuite la notion de "rhapsodie» appliquée au poème pour faire de l'Iliade un centon, en s'appuyant notamment sur les auteurs antiques. Dans la seconde partie des Conjectures, il examine le texte même : les incohérences internes, le comportement excessif des dieux comme des héros, les répétitions, les différences de tons... ne peuvent s'expliquer que par l'existence de plusieurs poètes aux desseins différents dont les textes ont été rassemblés par un compilateur. Selon lui, l'Iliade comprendrait «quarante tragédies» d'environ 400 vers qui ensuite, «cousues 
ensemble ", auraient donné naissance à l'épopée que nous connaissons. Aubignac relit en quelque sorte l'épopée à la lumière de la tragédie.

4 La méthode de l'abbé d'Aubignac consiste à appliquer les procédés d'analyse des contemporains à l'Iliade, et plus particulièrement des concepts universels plus ou moins hérités d'Aristote, même si ce dernier n'est pas cité : il s'appuie sur le bon sens et l'analyse de la totalité de l'œuvre. L'argumentation repose en grande partie sur la notion de cohérence textuelle et $\mathrm{G}$. Lambin fait à juste titre référence au concept normatif de la fonction auteur développé et théorisé bien plus tard par Michel Foucault. Pour Lambin (p.72), Aubignac allait contre le courant d'une époque qui privilégie l'ordre, le respect des règles et de l'autorité. Ce théoricien volontiers dogmatique, ce législateur des lettres fut selon lui un révolutionnaire plus d'un siècle avant la Révolution. Il s'agit du texte d'un esprit libre avant l'heure. Lambin exagère peut-être quand il voit dans la date de publication (1715) un lien avec la mort de Louis XIV.

5 Le texte de l'abbé n'est cependant pas exempt de faiblesses que G. Lambin rappelle. La forme, dont nous avons déjà souligné les lacunes, témoigne d'une certaine désinvolture, tout particulièrement quand Aubignac cite le texte (il se trompe, il emprunte sans vérifier, se contente de sources de seconde main...). Certes, on sait depuis Victor Magnien qu'Aubignac a travaillé sur la traduction latine de l'Iliade de Jean de Sponde ou celle d'Andrea Divo retouchée par de Sponde car il ne connaissait pas bien le grec (p.54-66), mais en cela l'abbé ne se distingue guère de ses contemporains, qui préféraient la tradition indirecte des Anciens et notamment le relais latin : il s'agissait de gommer les défauts de l'original, de l'adapter au goût des Modernes. Antoine Houdar de la Motte ne fit pas autre chose à partir de la traduction de l'Iliade de Mme Dacier en 1714. La désinvolture des Conjectures, les approximations et les erreurs sont la preuve que nous sommes plus en présence " d'exercices d'esprit » que de science, à une époque où les mondanités occupaient le premier rang et où le statut de l'ouvrage scientifique n'était pas encore fixé. Cependant les Conjectures témoignent aussi de véritables lacunes: si la documentation sur les auteurs antiques est abondante, le travail des Alexandrins sur l'épopée homérique n'est pas même évoqué, ni le commentaire d'Eustathe. On pourrait se demander pourquoi. Les sources modernes auxquelles se réfère ouvertement l'abbé sont principalement Érasme, ce qui est réducteur comme le rappelle G. Lambin (p. 291), et l'aspect polémique d'un tel choix mériterait une analyse. Aubignac n'évoque pas ouvertement la question de l'écriture et n'exploite pas le problème de l'usage de l'oralité. Il confond l'aède et le rhapsode, les Homérides et les Homéristes, et n'aborde jamais le texte de l'Iliade dans une perspective historique. Même la question de la mise par écrit du poème ne lui permet pas d'évoquer les différentes versions rivales ni l'aspect politique de la fixation du texte, notamment sous les Pisistratides à Athènes. Si G. Lambin s'applique à rappeler ces éléments majeurs pour mesurer l'apport des Conjectures, il ne le fait pas toujours de manière exhaustive et ne mentionne pas les travaux déterminants de G. Nagy sur l'histoire du texte homérique. Cette approche, même s'il n'en partage pas les conclusions, constitue cependant un élément important dans l'actualisation de la question homérique. Bref, G. Lambin fournit ici une édition critique de qualité et un outil scientifique de premier ordre dont l'introduction et la conclusion offrent une mise en perspective stimulante, mais parfois un peu orientée, oubliant une partie des relectures homériques actuelles. Le grand mérite de cette édition est de rappeler qu'à l'époque où les Conjectures furent prononcées (probablement pour l'académie fondée par Aubignac, dite des Belles 
Lettres, et rivale de l'Académie française), c'est-à-dire dans un cadre de circonstance, le savoir ne se distinguait pas encore de la parole ni de la recherche des mondanités. En ce sens, ce texte témoigne « d'une certaine idée de la science » que G. Lambin s'applique bien à dévoiler.

\section{AUTEURS}

\section{SYLVIE ROUGIER-BLANC}

Université de Toulouse (UTM)

rougier-blanc@neuf.fr 